Wright State University

\title{
CORE Scholar
}

International Symposium on Aviation

International Symposium on Aviation

Psychology - 2013

Psychology

2013

\section{Aviation Safety Evaluation by Wavelet Kernel-Based Support Vector Machine}

Chang Ming

You Xuqun

Follow this and additional works at: https://corescholar.libraries.wright.edu/isap_2013

Part of the Other Psychiatry and Psychology Commons

\section{Repository Citation}

Ming, C., \& Xuqun, Y. (2013). Aviation Safety Evaluation by Wavelet Kernel-Based Support Vector Machine. 17th International Symposium on Aviation Psychology, 560-566.

https://corescholar.libraries.wright.edu/isap_2013/21

This Article is brought to you for free and open access by the International Symposium on Aviation Psychology at CORE Scholar. It has been accepted for inclusion in International Symposium on Aviation Psychology - 2013 by an authorized administrator of CORE Scholar. For more information, please contact library-corescholar@wright.edu. 


\title{
Aviation safety evaluation by wavelet kernel-based support vector machine
}

\author{
Chang ming, You Xuqun \\ *School of Psychology, Shaanxi Normal University,Xian 710062,China, \\ cchangmingg@163.com
}

\begin{abstract}
In order to obtain the excellent evaluation effects, the wavelet kernel function is used as the kernel function of support vector machine,and the model is defined as wavelet kernel-based support vector machine.Thus, wavelet kernel-based support vector machine is applied to aviation safety evaluation. The two dimensional input vector of the training samples is employed to construct the training samples.The traditional radial basis function kernel-based support vector machine is used to compare with the wavelet kernel-based support vector machine.The testing results show that the evaluation error of the wavelet kernel-based support vector machine belongs to the range from 0.015 to 0.04 ,and the evaluation error of the traditional radial basis function kernel-based support vector machine belongs to the range from 0.02 to 0.07.Then,we can conclude that aviation accidents evaluation accuracy of wavelet kernel-based support vector machine is higher than those of traditional radial basis function kernel-based support vector machine.
\end{abstract}

Keywords: wavelet kernel; evaluation;aviation safety;support vector machine

\section{Introduction}

Recently, artificial neural networks have been used less in engineering field. Instead of artificial neural networks, support vector machine has been applied for various domains, due to its excellent generalization ability[1-6].Support vector machine proposed by Vapnik is a novel machine learning algorithm based on statistical learning theory[7,8]. Originally,support vector machine was developed to solve the classification problems. Recently, the method has been extended to solve the regression and evaluation problems. The choice of kernel function of support vector machine has a certain influence on the evaluation effects.In order to obtain the excellent evaluation effects, the wavelet kernel function is used as the kernel function of support vector machine, and the model is defined as wavelet kernel-based support vector machine.Thus, wavelet kernel-based support vector machine is applied to aviation safety evaluation. Aviation accidents from 1995 to 2003 in China are employed as our experimental data to test and analysis for aviation accidents[9] evaluation by using the proposed method.The two dimensional input vector of the training samples is employed to construct the training samples. The traditional radial basis function kernel-based support vector machine is used to compare with the wavelet kernel-based support vector machine.The testing results show that the evaluation error of the wavelet kernel-based support vector machine belongs to the range from 0.015 to 0.04 , and the evaluation error of the traditional radial basis function kernel-based support vector machine belongs to the range from 0.02 to 0.07. Then,we can conclude that aviation accidents evaluation accuracy of wavelet kernel-based support vector machine is higher than those of traditional radial basis function kernel-based support vector machine. 


\section{Wavelet Kernel-based Support Vector Machine}

Support vector machine proposed by Vapnik is a novel machine learning algorithm based on statistical learning theory.Recently, the method has been extended to solve the regression and evaluation problems[10,11].

Given a set of the training samples $\left\{\left(x_{i}, y_{i}\right)\right\}_{i=1}^{n}$, where $x_{i} \subset R^{m}$ denotes the input vector and $y_{i} \subset R$ denote the corresponding output. The evaluation function of support vector machine employs the following formula:

$$
y(x)=\omega \cdot \phi(x)+b
$$

where $\omega$ denotes the weight vector and $b$ denotes the bias.

In order to gain the values of the weight vector $\omega$ and the bias $b$, two positive slack variables $\xi, \xi^{*}$ are introduced,and infeasible constraint of the optimization problem is given as follows:

Minimize

$$
\frac{1}{2}\|\omega\|^{2}+C \sum_{i=1}^{n}\left(\xi_{i}+\xi_{i}^{*}\right)
$$

Subject to

$$
\begin{cases}y_{i}-\left\langle\omega, \phi\left(x_{i}\right)\right\rangle-b \leq \varepsilon+\xi_{i} & \xi_{i} \geq 0 \\ \left\langle\omega, \phi\left(x_{i}\right)\right\rangle+b-y_{i} \leq \varepsilon+\xi_{i}^{*} & \xi_{i}^{*} \geq 0\end{cases}
$$

where $C$ denotes the penalty.

Then, the Lagrangian multipliers $a_{i}, a_{i}^{*}$ are introduced to obtain the constrained optimization problem:

Maximize

$$
\sum_{i=1}^{n} y_{i}\left(a_{i}-a_{i}^{*}\right)-\varepsilon \sum_{i=1}^{n}\left(a_{i}+a_{i}^{*}\right)-\frac{1}{2} \sum_{i, j=1}^{n}\left(a_{i}-a_{i}^{*}\right)\left(a_{j}-a_{j}^{*}\right) K\left(x_{i}, x_{j}\right)
$$

Subject to

$$
\left\{\begin{array}{c}
\sum_{i=1}^{n}\left(a_{i}-a_{i}^{*}\right)=0 \\
0 \leq a, a^{*} \leq C
\end{array}\right.
$$

where $K\left(x_{i}, x_{j}\right)\left(K\left(x_{i}, x_{j}\right)=\phi\left(x_{i}\right) \phi\left(x_{j}\right)\right)$ denotes the kernel function.

Finally, the evaluation function of support vector machine is given as follows:

$$
y(x)=\sum_{i=1}^{n}\left(a_{i}-a_{i}^{*}\right) K\left(x_{i}, x\right)+b
$$


In this study, the wavelet kernel function is used as the kernel function of support vector machine, the wavelet kernel function can be shown as follows:

$$
k\left(x, x^{\prime}\right)=\prod_{i=1}^{M} \mathrm{H}\left(\frac{x_{i}-b}{a_{i}}\right) \mathrm{H}\left(\frac{x_{i}^{\prime}-b^{\prime}}{a_{i}}\right)
$$

where $X_{i}$ denotes the variable vector.

\section{Experimental Testing and Analysis}

As shown in Fig.1,aviation accidents from 1995 to 2003 in China are employed as our experimental data to test and analysis for aviation accidents evaluation by using the proposed method. The two dimensional input vector of the training samples is employed to construct the training samples. The traditional radial basis function kernel-based support vector machine is used to compare with the wavelet kernel-based support vector machine.As shown in Fig.2,the evaluation curve of the wavelet kernel-based support vector machine is given, the evaluation curve of the wavelet kernel-based support vector machine is near to the curve composed of the actual values. As shown in Fig.3, the evaluation error of the wavelet kernel-based support vector machine belongs to the range from 0.015 to 0.04 .

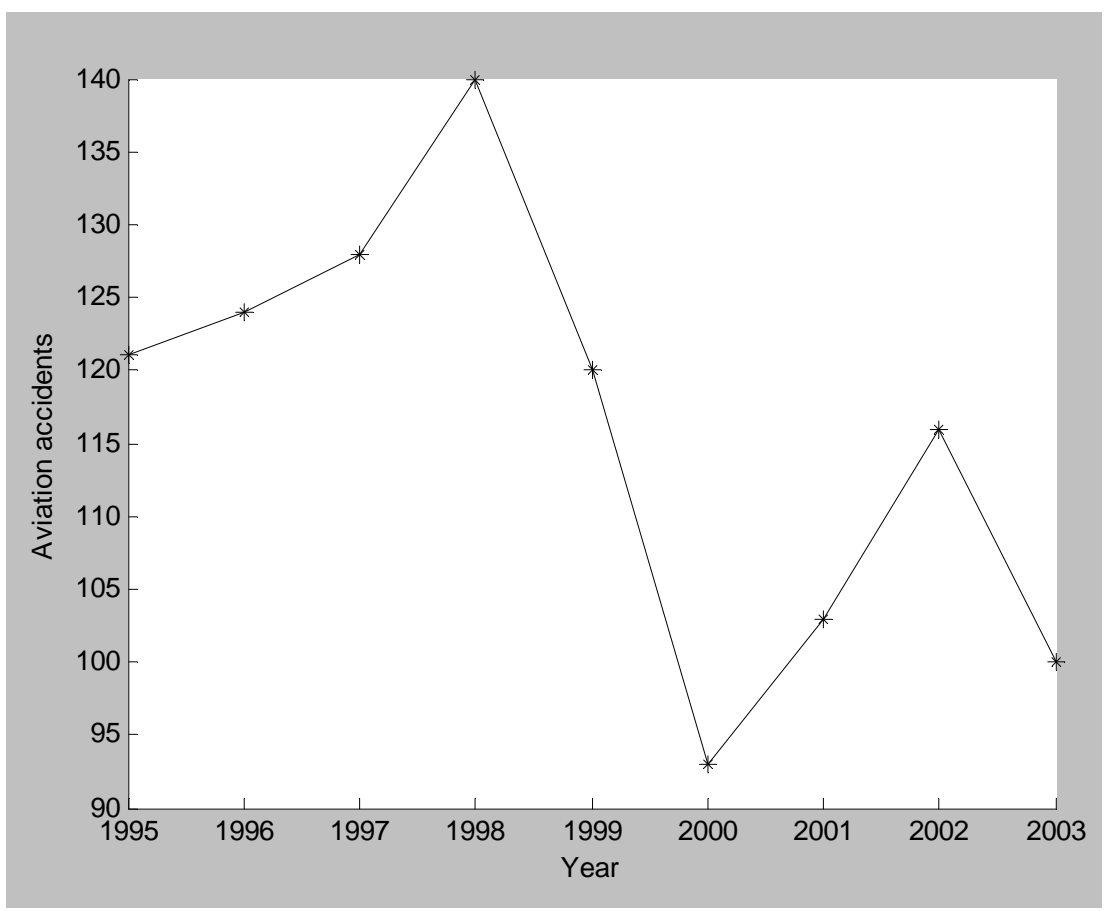

Figure 1. Aviation accidents from 1995 to 2003 in China 


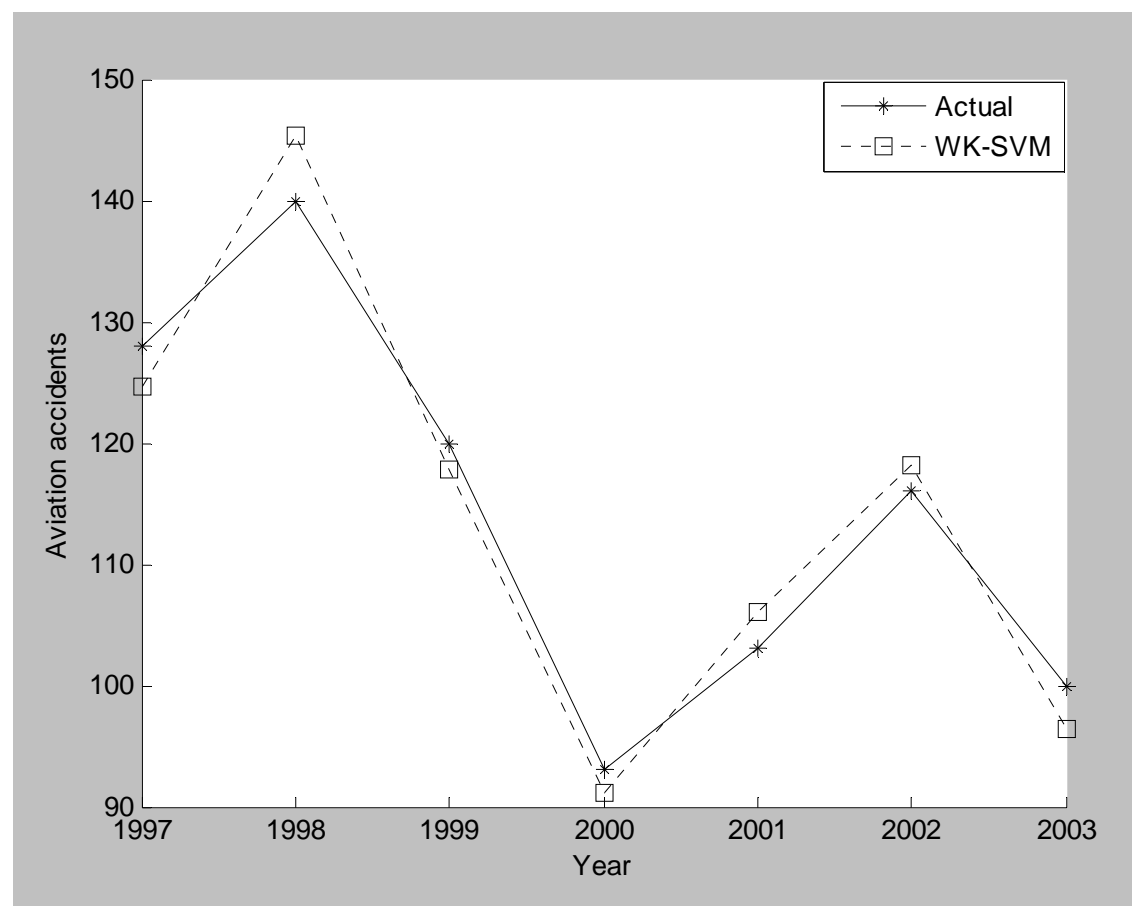

Figure 2. The evaluation curve of the wavelet kernel-based support vector machine

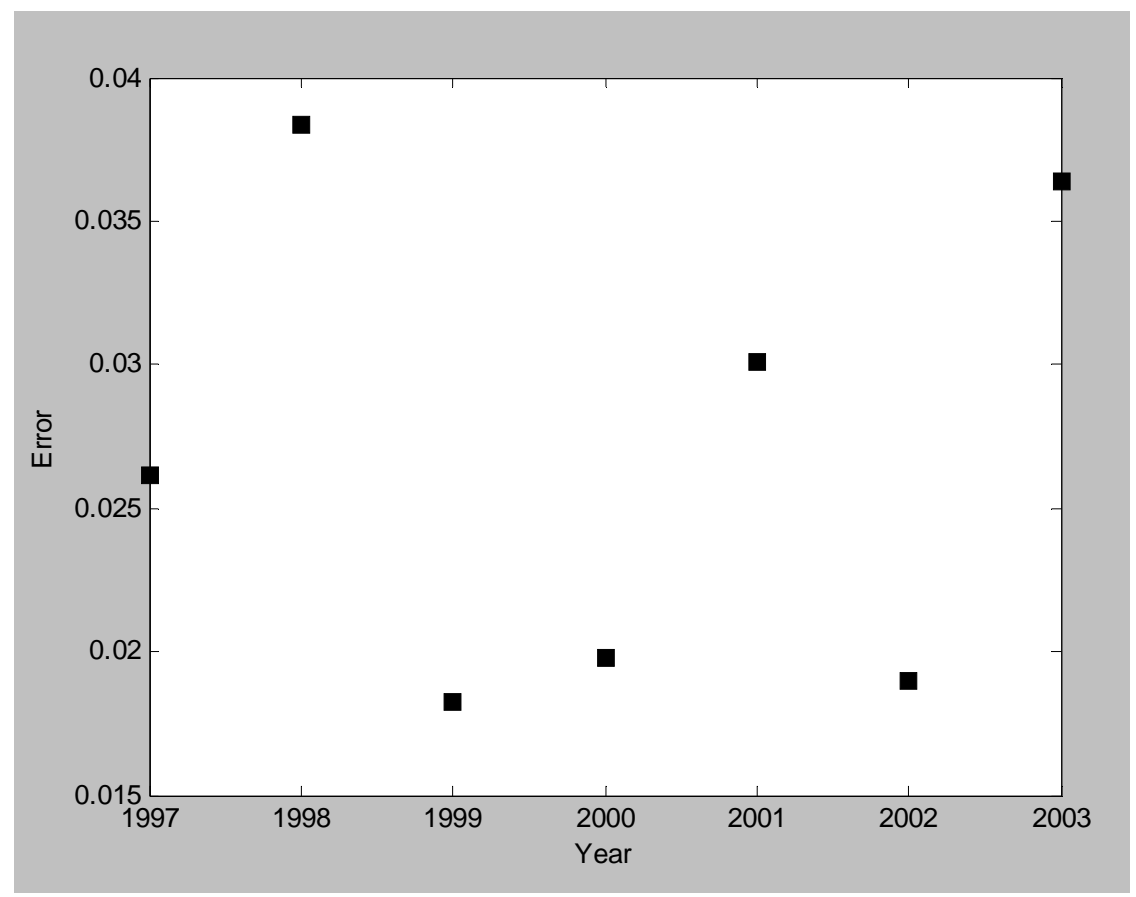

Figure 3. The evaluation error of the wavelet kernel-based support vector machine 


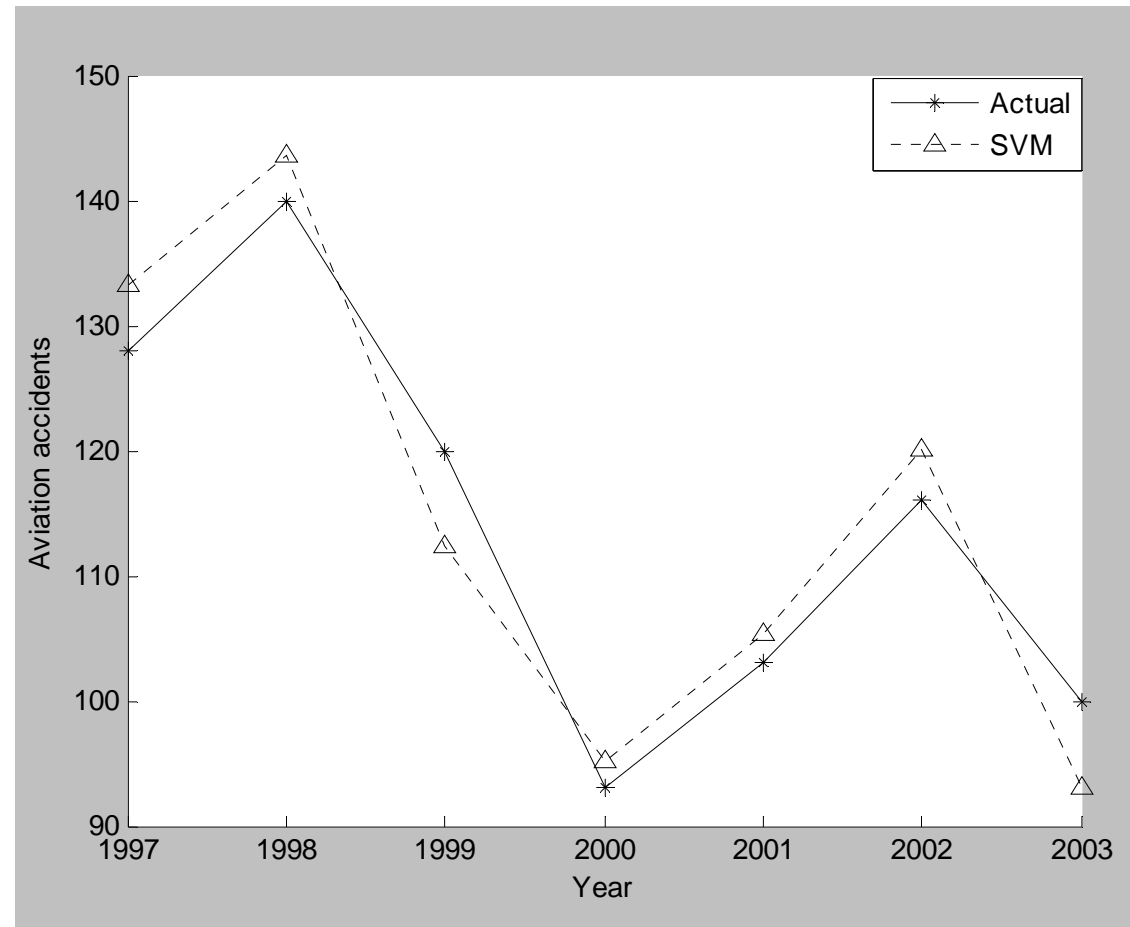

Figure 4. The evaluation curve of the traditional radial basis function kernel-based support vector machine

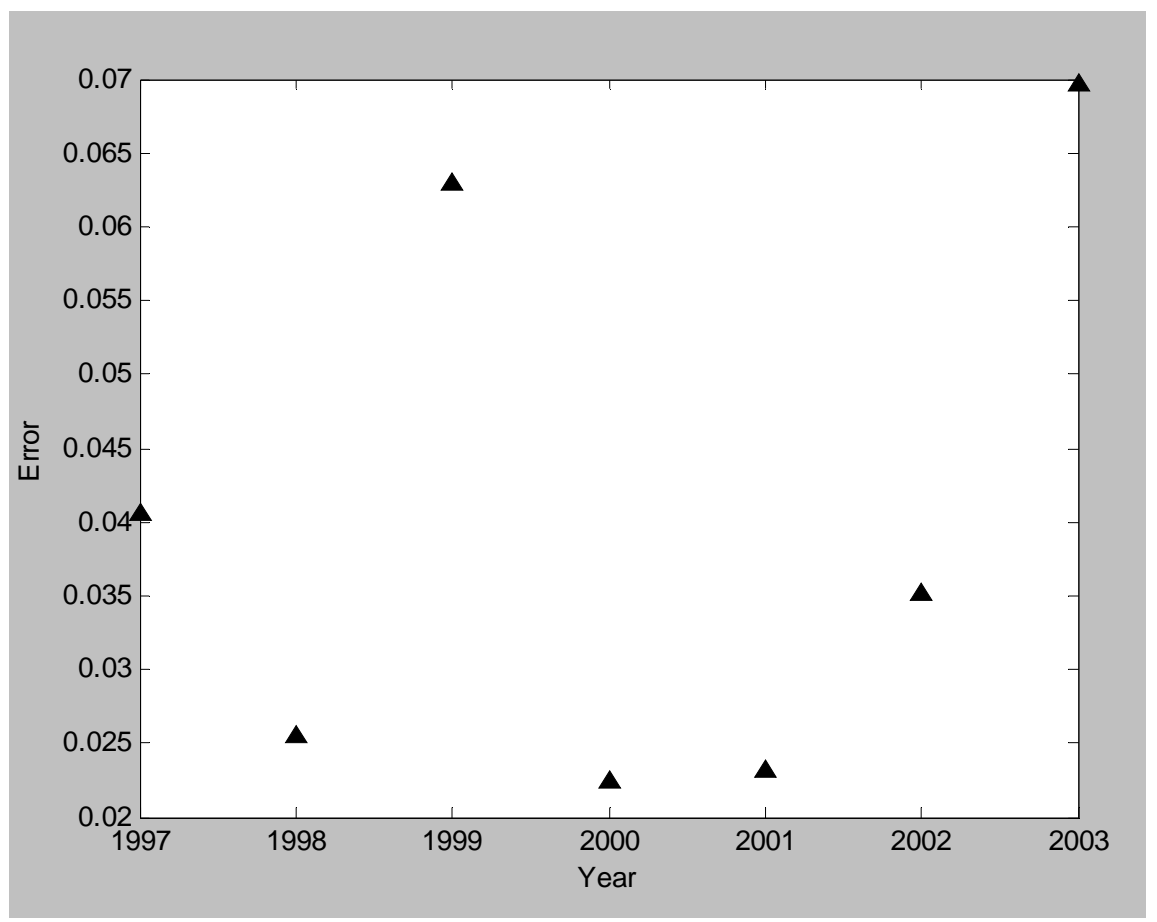

Figure 5. The evaluation error of the traditional radial basis function kernel-based support vector machine 


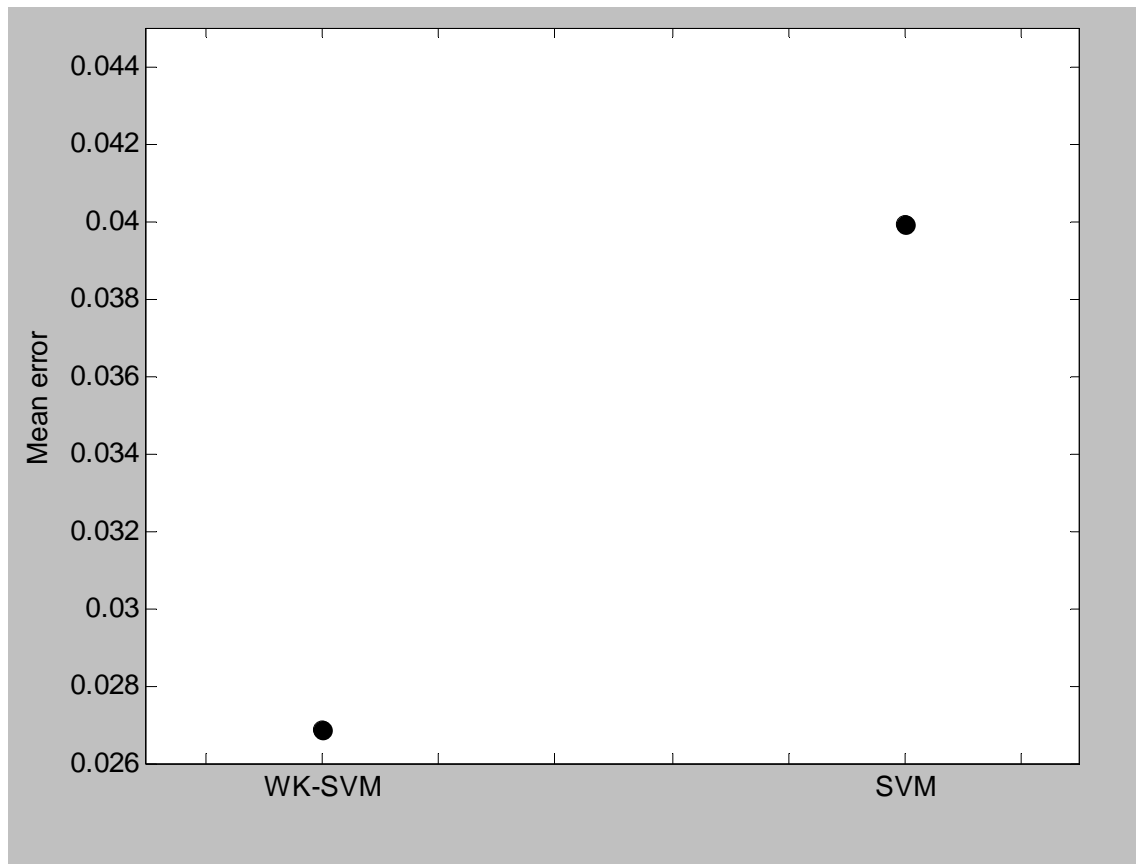

Figure 6. The comparison of the mean error between traditional radial basis function kernel-based support vector machine and wavelet kernel-based support vector machine

As shown in Fig.4, the evaluation curve of the traditional radial basis function kernel-based support vector machine is given, the evaluation curve of the traditional radial basis function kernel-based support vector machine is near to the curve composed of the actual values. As shown in Fig.5, the evaluation error of the traditional radial basis function kernel-based support vector machine belongs to the range from 0.02 to 0.07 .

As shown in Fig.6,the comparison of the mean error between traditional radial basis function kernel-based support vector machine and wavelet kernel-based support vector machine is given,which indicates that aviation accidents evaluation accuracy of wavelet kernel-based support vector machine is higher than those of traditional radial basis function kernel-based support vector machine.

\section{Conclusion}

Wavelet kernel-based support vector machine is applied to aviation safety evaluation. The wavelet kernel function is used as the kernel function of support vector machine, and the two dimensional input vector of the training samples is employed to construct the training samples. The traditional radial basis function kernel-based support vector machine is used to compare with the wavelet kernel-based support vector machine.The testing results indicate that aviation accidents evaluation accuracy of wavelet kernel-based support vector machine is higher than those of traditional radial basis function kernel-based support vector machine.

\section{References}

[1] Vahid Khatibi.B, Dayang N. A. Jawawi, Siti Zaiton Mohd Hashim, Elham Khatibi, "Neural Networks for Accurate Estimation of Software Metrics", IJACT, Vol. 3, No. 10, pp. 54 66, 2011. 
[2] Pierre E. ABI-CHAR, Bachar EL-HASSAN, Abdallah MHAMED, "An Enhanced Authenticated Key Agreement Protocol with a Neural Network-based Model for Joining-Phase in Mobile Environments", IJEI, Vol. 2, No. 2, pp. $103 \sim 112,2011$.

[3] K Lamamra, K Belarbi, "Comparison of Neural Networks and Fuzzy Logic Control Designed by Multi-objective Genetic aWlgorithm", IJACT, Vol. 3, No. 4, pp. 137 143, 2011.

[4] Luo Shihua, Jia Li, "Silicon Content Prediction Using the Hybrid Model by Fuzzy C-means Clustering and Artificial Neural Networks", AISS, Vol. 3, No. 8, pp. 78 84, 2011.

[5] Jinxue Sui, , Li Yang, Zhilin Zhu, "Flame Burning Condition Recognition Based on Improved ART Neural Network", IJACT, Vol. 3, No. 4, pp. $32 \sim$ 40, 2011.

[6] Ignacio Yélamos, Gerard Escudero, Moisès Graells, Luis Puigjaner, "Performance assessment of a novel fault diagnosis system based on support vector machines", Computers \& Chemical Engineering, Vol. 33, No. 1, pp.244 255, 2009.

[7] Haldun Aytuğ, Serpil Sayın, "Using support vector machines to learn the efficient set in multiple objective discrete optimization ",European Journal of Operational Research, Vol.193, No. 2, pp.510 519,2009.

[8] Enrique Romero, René Alquézar, "Comparing error minimized extreme learning machines and support vector sequential feed-forward neural networks",Neural Networks, Vol. 25, pp.122 $\sim 129,2012$.

[9] Qian WEI, Jingsha HE, , Xing ZHANG, "A User Admission Scheme for Civil Aviation Emergency Mesh Networks ", JDCTA, Vol. 5, No. 6, pp. $275 \sim 281,2011$.

[10] Tony Bellotti, Roman Matousek, Chris Stewart,"A note comparing support vector machines and ordered choice models' predictions of international banks' ratings",Decision Support Systems, Vol. 51, No.3, pp.682 687,2011.

[11] N. Shojai Kaveh, F. Mohammadi, S.N. Ashrafizadeh, "Prediction of cell voltage and current efficiency in a lab scale chlor-alkali membrane cell based on support vector machines ", Chemical Engineering Journal, Vol. 147, No.2-3, pp.161 172, 2009. 